TP Periodica Polytechnica Electrical Engineering and Computer Science

59(1), pp. 18-25, 2015

DOI: 10.3311/PPee. 7865

Creative Commons Attribution (i)

RESEARCH ARTICLE

\section{Young's Modulus and Energy Dissipation Determination Methods by AFM, with Particular Reference to a Chalcogenide Thin Film}

\author{
Judit Kámán ${ }^{1 *}$
}

Received 10 December 2014; accepted after revision 20 January 2015

\begin{abstract}
There are two main ways to investigate mechanical properties by atomic force microscopy (AFM): force curve measuring and tapping-mode phase mapping. This paper provides an overview of these techniques with special regards to the determination of Young's modulus from force curves and the calculation of tip-sample energy dissipation from phase maps.

Besides, both methods were used to study the mechanical properties of amorphous chalcogenide thin film samples. As ${ }_{20} \mathrm{Se}_{80}$ thin film was prepared by thermal evaporation on a glass substrate and a He-Ne laser (633 nm) was used to prepare a holographic grating on the thin film.

The obtained results showed significant difference in the Young's modulus between the peaks and the valleys and contrast was also observed on the dissipation map which can be connected to the variation of mechanical properties in accordance with the grating.
\end{abstract}

\section{Keywords}

AFM, Young's modulus, energy dissipation, chalcogenide
${ }^{1}$ Department of Electronics Technology,

Faculty of Electrical Engineering and Informatics,

Budapest University of Techology and Economics,

H-1111 Budapest, 18 Egry József street, Hungary

*Corresponding author, e-mail: nemcsakhanemis@gmail.com

\section{Introduction}

Atomic Force Microscopy (AFM) is a versatile tool for studying surfaces at the nanoscale level. Since its invention - 1986 by Binnig at al. [1] - the number of the possible applications of the AFM has increased significantly beyond the classical topography mapping. Nowadays, it is also used for nanofabrication (or nanolithography) [2, 3], the investigation of the physical and mechanical properties of the sample, and the identification of material variations. The aim of this paper is to present two methods for the determination of surface mechanical properties, and illustrate these methods through measurements on chalcogenide thin film gratings. Young's modulus can be obtained from the contact-mode point-spectroscopy curve, which in general is called the force-curve. This gives information about the tip-sample interaction at a single point of the surface, thus, describing the variation of material properties at an area with this technique is very time-consuming. Recent improvements in AFM instrumentation (e. g. Bruker's Force Peak $\mathrm{QNM}^{\circ}$ ) aim to provide elastic information about the surface for a whole scanned area at high resolution and with a scanning speed similar to topography mapping scan-rate. In general these novel techniques require special equipment.

However other material properties of the whole area of the sample can be investigated with a basic AFM by using the tapping-mode phase map. At tapping-mode the force between the tip and the sample cannot be studied directly, but the average energy dissipation can be calculated based on the model of Cleveland at al. [4]. Anczykowski et al. [5] have developed complementary equipment for microscopes, which allows the direct mapping of energy dissipation.

Young's modulus and energy dissipation determination methods were used on illuminated $\mathrm{As}_{20} \mathrm{Se}_{80}$ chalcogenide thin films. It is known that illumination of the amorphous chalcogenide thin films with bandgap light can lead to significant optical and structural transformations, which can be used to create for example holographic gratings on the films. Although these photo-induced changes are studied in great detail [6], there is little information concerning the elastic/hardness properties of the illuminated material. We hope that through the application 
of these two methods we can describe the mechanical properties of the illuminated chalcogenide films.

\section{Determination of Young's modulus}

\subsection{Analysis of the force-distance curve}

Contact-mode point-spectroscopy is an AFM technique to characterize the tip-sample interaction force versus the tipsample distance, while the tip moves down and up at one point. The information contained by these force curves can refer to several material properties such as Young's modulus, adhesion, deformation and dissipation. However, it can be difficult to gain accurate quantities.

The total force between the tip and the sample may consist of several components, such as adhesion force, short-range chemical force (which can be described by Lennard-Jones potential), electrostatic and magnetic forces.

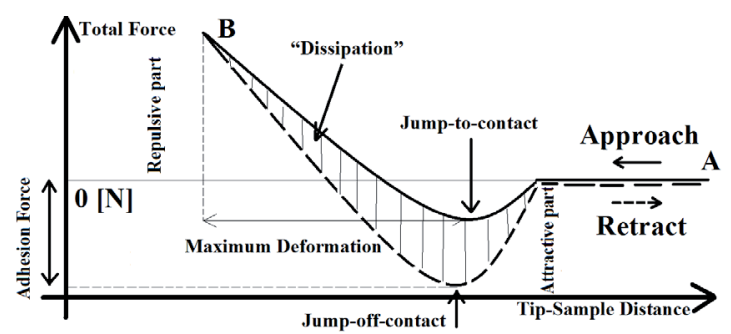

Fig. 1 Typical force-distance curve, where the apostrophe around the "Dissipation" denote that the whole striped area is not necessarily only the work of the dissipation, some parts can be derived from adhesion work

Figure 1 presents a typical force-distance curve. At the beginning of the point-spectroscopy the tip is far from the surface (see point A on Fig. 1) and there is negligible or no interaction between them. As the probe moves towards the surface, it gets in the field of the attractive forces like van der Waals, electrostatic or capillary forces. At some tip-sample distance the attractive forces overcome the cantilever stiffness and the tip jumps into contact with the surface. Then the tip stays on the surface and as the loading force increases on the probe, the cantilever deflection also increases and the sample is pressed. The probe then starts to withdraw to its initial position. During the unloading process the force decreases and reaches a point when the tip "jumps off” from surface. The force which belongs to this point can be identified as an adhesion force between the tip and the surface.

If there is no plastic deformation produced by the pressing of the tip the hysteresis between the repulsive parts of the loading- unloading curve almost disappears, only the adhesion term appears. Thus the area above the retracting curve and under the zero force line (see Fig. 1) can be referred to as the work of adhesion [7].

In case of a plastic deformation - which is the most common occurrence - the area of the hysteresis loop is related to the dissipation but it is difficult to separate it from the effect of the adhesion.

We can evaluate the maximum sample deformation which consists both the plastic and the elastic deformation. It can be determined as the distance between the point B on Fig. 1 where the loading force has a maximum - and the point where the tip first reaches the surface (jump-to-contact point).

At the repulsive region the bend of the approaching and withdrawing curves testify that the tip has indented the sample. Several models were published which describe the contact mechanics such as Hertz [8], JRK (Johnson-Roberts-Kendall model) [9] or DMT (Derjaguin-Muller-Toporov model) [10]. At first approximation, the surface forces are negligible so we applied the model introduced by Hertz and modified by Sneddon $[11,12]$ and we used it for the approaching curve.

\subsection{Determination method of Young's modulus}

As we mentioned above, the contact-mode force-distance curves carries information about the elastic properties of the sample.

However, in practice, the AFM does not measure the direct force or distance of the tip-sample. Furthermore, depending on the measuring equipments in general, one end of the cantilever is fixed and the sample holder can be moved by a piezo crystal. The raw data obtained from the contact-mode point-spectroscopy are the (absolute) deflection of the cantilever $(d(z))$ and the piezo position of the sample $(z)$ while the tip approaches and leaves the surface. To calculate the force and tip-sample distance, the deflection $(D(z))$ which is relative to the force-free cantilever deflection (in A point at Fig. 1) and the piezo movement $(Z)$ are needed. It means that there are the offsets $\left(z_{0}, d_{0}\right)$, that must be subtracted from the measured data; we will discuss later how to establish these.

It can be assumed that the cantilever is linearly elastic with the $k$ spring constant, thus the deflection $(D(z))$ can be converted to the corresponding force $(F)$ using Hook's law:

$$
F=k \cdot D(z)
$$

The distance $(\Delta)$ between the tip and the surface can be written as a difference of the piezo movement and the deflection:

$$
\Delta=Z-D(z)
$$

To determine Young's modulus, we assume that from the point when the tip reaches the surface the sample deformation is elastic. Increasing the loading force exerted by the cantilever, the material deformation will be irreversible and our model will become invalid.

Choosing the correct spring constant of the probe with regards to the material of the sample is essential to determine the Young's modulus. Using a cantilever with too large or too small elasticity, at the repulsive part (see Fig. 1) the bend of the force curve would not appear, instead, only a straight line would be seen. 
To gain the elastic modulus from the measured approaching curves, we employed the Hertzian model modified by Sneddon. It describes the elastic indentation of a soft sample by a stiff cone $[11,12]$. This model relates the applied force $(F)$ to the indentation depth $(\delta)$ through Young's modulus $(E)$. Since the cantilever spring is linear for a small deflection, the Hook's law provides the connection between the cantilever deflection $(D(z))$ and the applied loading force of the indentation:

$$
k D=\frac{2 E \tan (\alpha)}{\pi\left(1-v^{2}\right)} \delta^{2}
$$

where $v$ is the Poisson ratio and $\alpha$ is the half opening angle of the tip.

On an infinitely stiff sample the piezo movement is equal to the cantilever deflection $D(z)$, while on a soft sample the latter decreases by the indentation depth $\delta$. Since the microscope measures the absolute piezo position and cantilever deflection data ( $z$ and $d(z)$ ), for the calculations the offsets $z_{0}$ and $d_{0}$ have to be defined. Thus the indentation $\delta$ is given by the following equation:

$$
\delta=\left(z-z_{0}\right)-\left(d-d_{0}\right)=Z-D
$$

The $z_{0}$, which is commonly called contact point (CP), is the vertical position of the piezo where the probe first makes contact with the sample. Identifying CP is critical for precise fitting. Despite several papers were published on this issue, currently there is no widely-accepted default method for its determination. The simplest way to define $\mathrm{CP}$ is the visual assessment, although, the problem with this method is, that it can be time consuming, subjective and relatively hard to reproduce. The difficulty to algorithmically find the point where the deflection begins to increase depends on the shape of the force curve, which is defined by the interaction between the tip and the surface.

In the case of most biological samples the attractive part of this curve is missing, instead, the first part of the curve is straight. In this case the definition of the CP could be problematic even though several studies presented algorithms for this [13-15].

When the attractive part of the force curve appears (see Fig. 1) the determination of the CP point is less complicated according to our measurements. In this case we can assume that the minimum of the force curve is equivalent to the $\mathrm{CP}$ point. Because the force curve is obtained by applying Eq. (1) and (2) on the raw data, the CP point is the minimum of the absolute deflection $(d(z))$ versus piezo position $(z)$ curve too. So we shifted the origin of the raw data curve to the minimum (thus the $d_{0}$ is determined too, but there may be some bias from the free cantilever deflection) and we assumed that the model described by Eq. (3) can be applied from this point. However, it is possible that the minimum is not a point, but a line segment. Because of this and because of the more accurate fitting we allow a little deflexion from the minimum point in the $z_{0}$ piezo position, and it is considered in the fitting equation through a new $c$ parameter, thus the new form of the deflection is:

$$
\delta=(Z-c)-D
$$

Substituting Eq. (5) in (3) and rearranging it gives:

$$
Z=c+D+\sqrt{\frac{k\left(1-v^{2}\right) \pi}{2 E \tan (\alpha)}} \sqrt{D}
$$

Based on this model two parameters can be obtained by fitting this equation on the $Z-D$ data, which are $c$ and the A-factor as defined by Eq. (7).

$$
A=\sqrt{\frac{k\left(1-v^{2}\right) \pi}{2 E \tan (\alpha)}}
$$

From this Young's modulus can be expressed as Eq. (8):

$$
E=\frac{k\left(1-v^{2}\right) \pi}{2 \tan (\alpha)} A^{-2}
$$

Figure 2 shows a typical deflection signal-piezo movement data with the fitted curve.

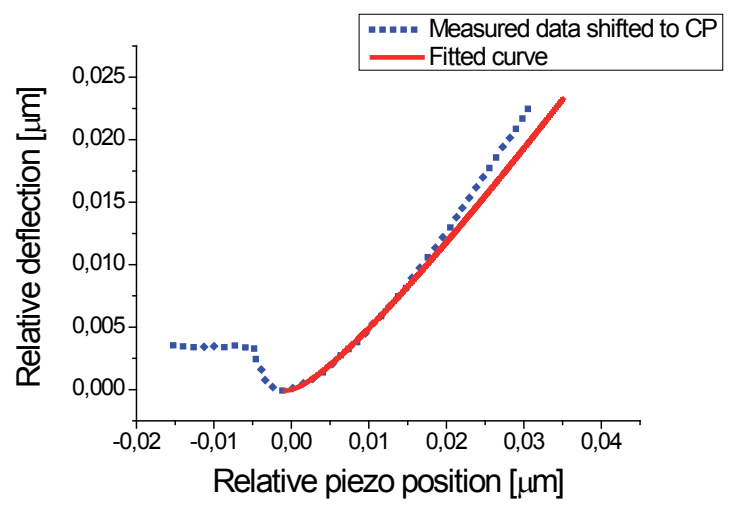

Fig. 2 Contact mode point-spectroscopy data (dotted line) shifted to the CP point and fitted curve (solid line) based on Eq. (6)

\section{Tip-surface energy dissipation}

Since in tapping-mode AFM the tip-sample interaction causes phase lag in the measured tapping signal of the cantilever compared to the drive, thus the phase-lag can characterize the material properties.

There are two common ways to investigate the connection between the phase-lag and the tip-surface interaction: through forces or energy.

From the viewpoint of force, in the most simplified case the tapping cantilever can be modelled as a one dimensional linear spring-mass system [16-18]. The equation of motion is a forced oscillation completed with a non-linear term which describes the tip-sample force. The most generally used terms are the van der Waals force modified according to the most common AFM 
geometries (see Table 3 in [19]) through the Hamaker constant. To solve this non-linear differential equation is generally complicated and numerical computations are needed.

To investigate material variations Cleveland et al. introduced a more efficient and practical method [4] based on the energy dissipation between the tip and the sample. The vantage of this model is that we do not need to make a priori prediction for the interaction force. The only assumption is that the steady-state response of the cantilever is nearly sinusoidal which is verified by their experiments. The model is based on the analysis of the energy flow in and out of the dynamic system per a cycle of tip motion. In equilibrium the average power fed into the cantilever by an external drive $\left(\left\langle P_{\text {in }}>\right)\right.$ must be converted to the average power dissipated by the motion of the cantilever beam in the medium (generally air) $\left(<P_{0}>\right)$ and by the tip-sample interaction $\left(<P_{t s}>\right)$ :

$$
\left\langle P_{\text {in }}\right\rangle=\left\langle P_{0}\right\rangle+\left\langle P_{t s}\right\rangle
$$

what we are interested in is the tip-surface dissipation $\left\langle P_{t s}\right\rangle$ and it can be expressed from Eq. (9), since the external excitation is known and the beam damping can be well modelled by viscous damping [5]. In order to calculate it we apply that the cantilever base position follows the external drive motion:

$$
z_{d}(t)=A_{d} \cos (\omega t)
$$

where $\omega$ is the angular frequency of the excitation and $A_{d}$ is the drive amplitude. Furthermore we exploit the assumption that the motion of the tip at the end of the cantilever is sinusoidal too:

$$
z(t)=A \cos (\omega t+\varphi)
$$

where $A$ is the amplitude of the cantilever and $\varphi$ is the phase lag compared to the drive. Using Hook's law the instantaneous power which can be delivered to the cantilever by the drive is the following:

$$
P_{\text {in }}(t)=F_{d}(t) \dot{z}_{d}(t)=k\left[z(t)-z_{d}(t)\right] \dot{z}_{d}(t)
$$

where $k$ is the spring constant of the cantilever.

Thus the average power input per oscillating cycle can be obtained by integrating over one period:

$$
\left\langle P_{i n}\right\rangle=\frac{\omega}{2 \pi} \int_{0}^{\frac{2 \pi}{\omega}} P_{i n}(t) d t=\frac{1}{2} k \omega A_{d} A \sin \varphi
$$

Since the dissipation by the motion can be described as a viscous damping characterized by the $\beta$ damping constant, the instantaneous power will be the following:

$$
P_{0}=F(t) \dot{z}(t)=\beta \dot{z}(t)^{2}
$$

Integrating over a cycle the average power yields:

$$
\left\langle P_{0}\right\rangle=\frac{\omega}{2 \pi} \int_{0}^{\frac{2 \pi}{\omega}} P_{0}(t) d t=\frac{1}{2} \beta A^{2} \omega^{2}
$$

Regularly during the tapping-mode AFM measurements the driven angular frequency is chosen near to the resonant angular frequency of the free cantilever which is close to the natural angular frequency:

$$
\omega_{0}=\sqrt{\frac{k}{m}}
$$

where the $m$ is the mass of the cantilever. For this reason we can express the $\beta$ damping coefficient and the $A_{0}$ free oscillation amplitude in experimentally accessible quantities:

$$
\begin{aligned}
& \beta=\frac{k}{Q \omega_{0}} \\
& A_{0}=Q A_{d}
\end{aligned}
$$

where $Q$ is the quality factor. $Q$ and $\omega_{0}$ can be determined from the measured free-cantilever resonance curve.

Using the above reductions and substituting Eq. (16) and Eq. (17) in Eq. (13) and Eq. (15), we gain a useful correlation:

$$
\left\langle P_{t s}\right\rangle=\frac{1}{2} \frac{k A^{2} \omega_{0}}{Q}\left[\frac{A_{0}}{A} \sin \varphi-1\right]
$$

Far from the surface the only effect that causes the dissipation is the viscous damping thus $\left\langle P_{t s}>=0\right.$. In this case Eq. (19) yields $\sin \varphi=A / A_{0}$. Furthermore this is a free oscillation in the air at $\omega \approx \omega_{0}$ angular frequency hence $A \approx A_{0}$. The consequence of the model is that $\varphi$ equals $90^{\circ}$ far from the sample where there is no energy loss. The domain of the $\varphi$ phase-lag is between $0^{\circ}$ and $180^{\circ}$ and any deviation from the $90^{\circ}$ indicates the presence of the tip-sample dissipation.

Rearranging Eq. (19) to express $\sin \varphi,\left\langle P_{t s}>\right.$ and $A$ are the only variables on the right side:

$$
\sin \varphi=\frac{A}{A_{0}}\left(\frac{2 Q}{k \omega_{0}} \frac{\left\langle P_{t s}\right\rangle}{A^{2}}+1\right)
$$

During tapping-mode imaging $A$ is kept constant so any alteration in the sinus of the phase-lag is due to the probe-sample dissipation. Thus contrast in the sinus of the phase map correlates to the contrast of the dissipation map presumably indicating the material property variations.

In order to apply this model to the phase map a preliminary tapping-mode point-spectroscopy measurement is needed to determine $A_{0}$ and the amount of the phase-lag which belongs to the free oscillation and to make it equal to $90^{\circ}$.

\section{Materials and Methods \\ 4.1 AFM measurements}

Atomic force microscope measurements were done with a Veeco (lately Bruker) diInnova type microscope in full contact- and tapping-mode with $512 \times 512$ sampling rate and $1 \mathrm{~Hz}$ scan rate. The PID values were optimized according to the user manual. We used Budget Sensors TAP 150Al-G probes for both 
contact- and tapping-mode point-spectroscopy and tappingmode imaging. The nominal properties of the probes were the following: cantilever spring constant $(k) 5 \mathrm{~N} / \mathrm{m}$, resonance frequency $\left(\omega_{0}\right) 135 \mathrm{kHz}$, half cone angle $(\alpha) 20^{\circ}$, quality factor $\left(Q_{\text {cant }}\right)$ 157. (Note that the actual values were obtained before every measurement during the tuning of the cantilever). For data evaluation the freeware Gwyddion 2.27 software was used.

\subsection{Sample preparation}

$\mathrm{As}_{20} \mathrm{Se}_{80}$ chalcogenide thin films were prepared by thermal evaporation (TE) onto a glass substrate at $5 \times 10^{-6} \mathrm{mbar}$ pressure. The composition of the samples was measured with energy dispersive X-ray spectroscopy (EDAX) using a Hitachi S-4300 system. The thickness of the samples was measured by an Ambios XP-1 profilemeter.

Holographic gratings were recorded by using two p-polarized and one additional s-polarized laser beams as this configuration has the most significant effect in chalcogenides [20]. For this purpose a He-Ne laser (633 nm) was used.

\section{Results and Discussions}

\subsection{Determination of Young's modulus}

Contact-mode point-spectroscopy measurements were done on the $\mathrm{As}_{20} \mathrm{Se}_{80}$ chalcogenide thin films to investigate its elastic properties. Figures 3 and 4 present the reference surface (prior to any illumination) and also the gratings caused by the illumination. Due to the smoothness of the reference area, it was scanned with $10 \mu \mathrm{m} \times 10 \mu \mathrm{m}$ scan-size, while the gratings was scanned on a $50 \mu \mathrm{m} \times 50 \mu \mathrm{m}$ area, as the periodicity of the grating is around $9.5 \mu \mathrm{m}$.

The force curves were obtained along the peaks and the valley bottoms in a row (in the $\mathrm{x}$ lateral dimension) by using the automatic position system of the scanner. The precise positioning of the AFM tip is essential, since the contact between the tip and the surface - the angle of incidence and the effective contact surface area - can greatly affect the force measurements. We consider the variation of contact, besides other effects, as a contributing factor to the deviation of the measurements. These other factors could be the possible contaminations on the surface and the irregularity of the gratings like the unequal depths of the valleys (see Fig 4 (C)).

Figure $5 \mathrm{~A}$ ) represents the measured deflection-distance curves at 10 peaks and 10 valleys points, which were shifted to their $\mathrm{CP}$ points. Despite the deviation of the curves the difference between the peaks and valleys seems to be significant. Figure 5 B) shows the A-factors resulting from the fittings of aforementioned curves. Assuming that the distributions of the A-factors of the peaks and valleys can be approximated by the normal distributions, we used Welch's t-test to examine their relation. It confirmed that there is a significant difference between the fitted parameters of peaks and of valleys at a significance level $(p)$ of 0.01 .
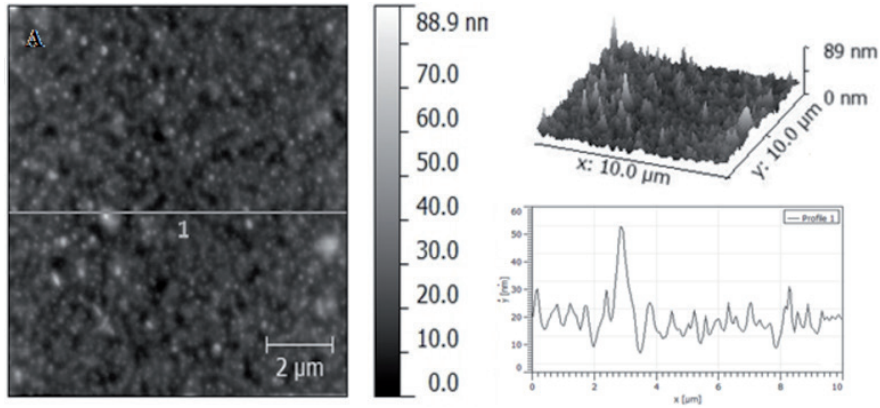

Fig. 3 A) Tapping-mode topography AFM image on the reference area of the chalcogenide thin film; B) is its $3 \mathrm{D}$ representation and C) is the corresponding cross-section at marker 1 .
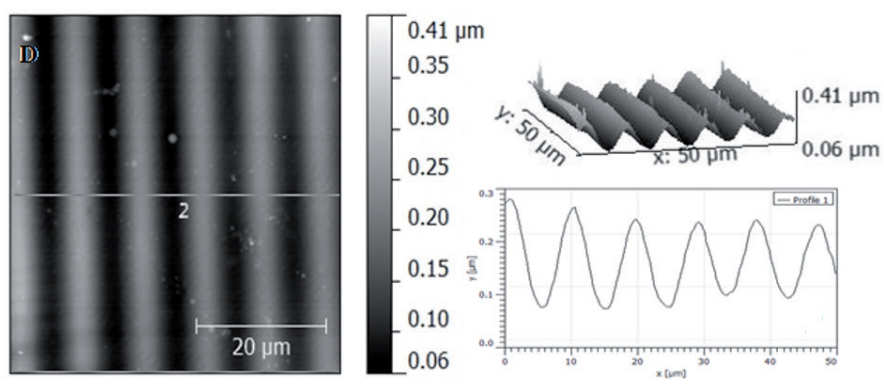

Fig. 4 A) Tapping-mode topography of the illuminated area; B) illustrates the gratings in $3 \mathrm{D} ; \mathrm{C}$ ) is the corresponding cross-section at marker 2.
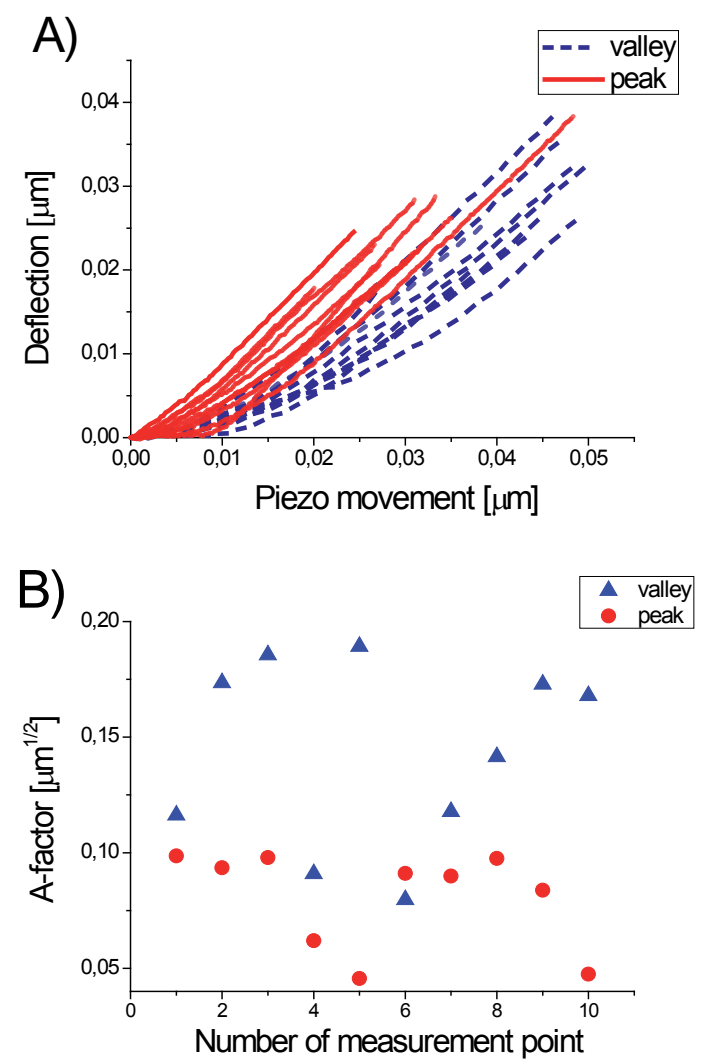

Fig. 5 A) Deflection-force curves of 10 peaks and 10 valleys points obtained from the contact-mode point-spectroscopy measurements and shifted to their CP points. B) The A-factors obtained by fitting on the curves of A). 


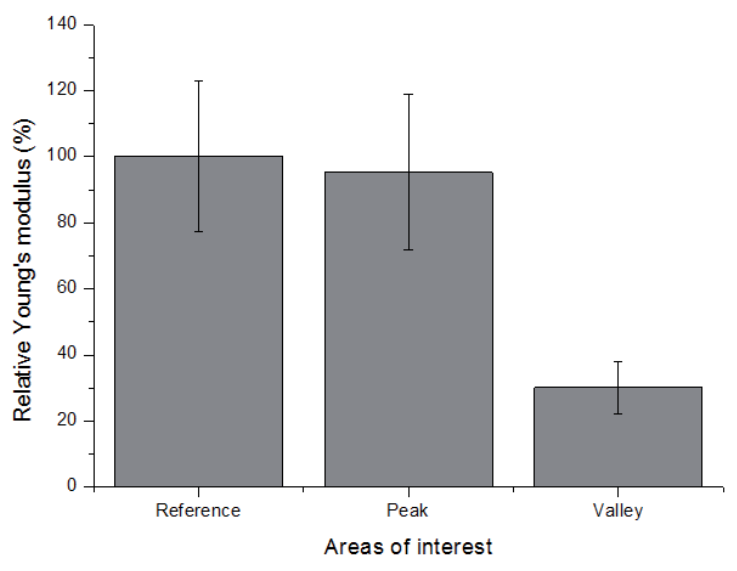

Fig. 6 Relative Young's modulus values calculated from the A-factors of Fig. 1. The relative values are compared to the mean of the reference area (which is $100 \%$ ). The number of averaged measurements are 16,10 and 10 for the reference, peaks and valleys, respectively

To determine Young's modulus $(E)$ from the fitted A-factor the precise knowledge or determination of the cantilever parameters are required. For our cantilevers the nominal spring constant $(k)$ is $5 \mathrm{~N} / \mathrm{m}$, but regarding the datasheet of the supplier the variation is between $1.5 \mathrm{~N} / \mathrm{m}$ and $15 \mathrm{~N} / \mathrm{m}$. Since we do not have a method to precisely determine the force constant of the cantilevers we refrain from calculating absolute modulus values. Instead, in Fig. 6 we present relative modulus values compared to the reference, which was measured on the chalcogenide surface, far from the illuminated area. It can be seen, that the average Young's modulus of the valleys is nearly the third of the peaks, which is close to the reference area. This means that the valleys are softer than the peaks, which can be explained by the photostructural transformations during the fabrication of the grating. Upon illumination, the structural deformation of the surface is caused by lateral mass transfer from the dark to the illuminated areas [21, 22]. The accumulation of the material in the peaks and, in the same time, the lack of the material in the valleys may cause local density and hardness increase/decrease along the surface.

\subsection{Tip-surface energy dissipation}

To be able to calculate the dissipation map of the surface tapping-mode point-spectroscopy measurements were done. From these curves it was possible to determine the phase-lag and $A_{0}$ as we discussed in the theory section. Figure 7 presents the amplitude and phase signals obtained at the reference area of the $\mathrm{As}_{20} \mathrm{Se}_{80}$ chalcogenide thin film and also the tip-surface dissipation, which was calculated by Eq. (19).

A slight rise is noticeable in the dissipation curve. It can be interpreted as a result of the increase in the indentation caused by the tip. During the indentation process the tip-sample effective contact area and therefore the friction force increase, which in turn causes increased dissipation [5].

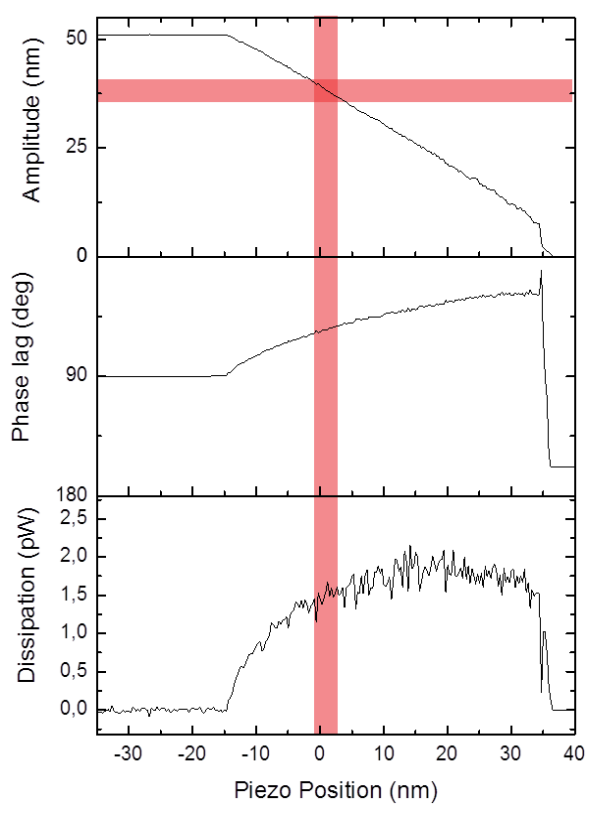

Fig. 7 A typical tapping-mode point-spectroscopy with the calculated tip-surface energy dissipation measured at the reference area of the sample. Red bars indicate the amplitude range during a closed loop amplitude-modulated scan.
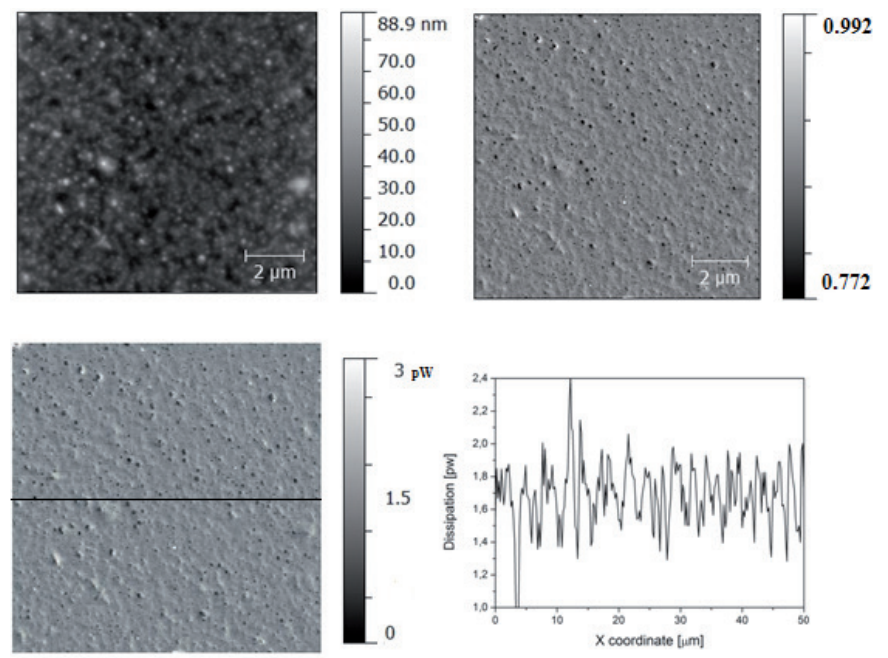

Fig. 8 A),tapping-mode AFM topography; B) sinus of the phase at the reference area; and C) the calculated dissipation map with the corresponding cross-section at the indicated marker (D).

The red bars indicate the range of the amplitude signal during a closed loop AM mode scan, while the feedback tries to maintain a constant amplitude and thus only minimal variation may occur. During our subsequent measurement we used an amplitude setpoint $\left(A_{s} \sim A\right)$ that our approximately constant $A$ / $A_{0}$ value was 0.8 . It can be seen that in this region (highlighted by the red bars) the calculated dissipation has small sensitivity to the variations in the amplitude, and thus we can presume that it will be primarily depending on the material properties of the surface.

Figure 8 presents the topography and the sinus of the phase map at the reference area and the calculated dissipation map by 
Eq. (19) using constant amplitude. The contaminations on the surface increase the standard deviation - as it can be seen on the cross section on the Fig. 8 D) - and may cause a little shift in the average dissipation. The presence of the contaminations increases the roughness of the surface which can influence the friction force and adhesions. For more details about the quantitative characterisation of surface structure and about the effect of the roughness see [23-25].
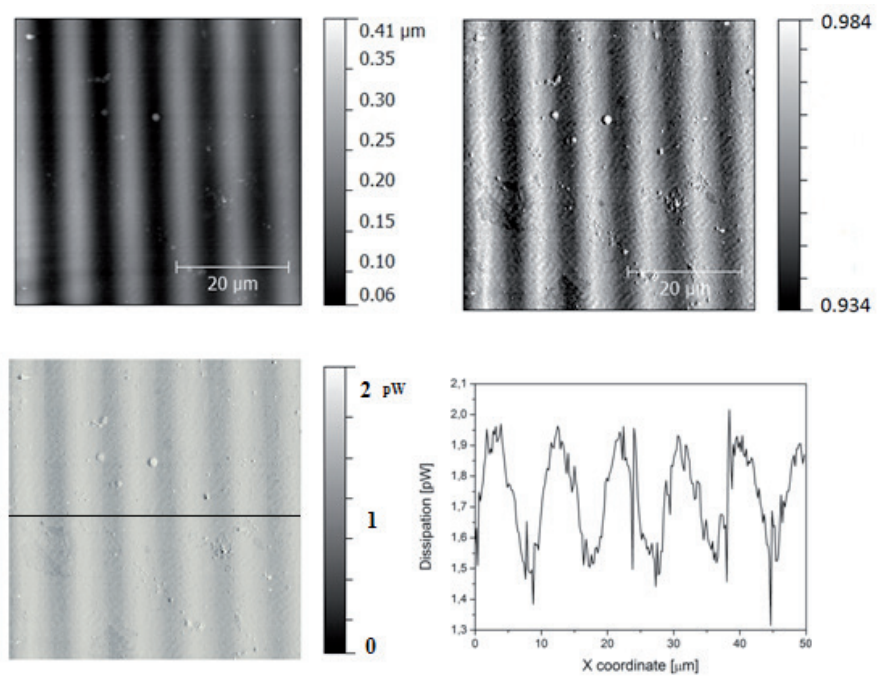

Fig. 9 A) tapping-mode AFM topography; B) the sinus of the phase on the gratings; and C) the calculated dissipation map with the corresponding cross-section at the indicated marker (D).

In Figure 9 B) the sinus of the phase map shows sharp contrast between the peaks and valleys. However, the dissipation is the result of the non-conservative forces contrary to the elasticity, the calculated dissipation map correlates with the previously obtained Young's modulus, namely the valleys have lower dissipation compared to the peaks. Figure 9 D) also presents a cross-section of the dissipation map, which shows distinct differences between the peaks and valleys.

The numerical value of the dissipation is strongly depends on the accuracy of determination of the $k$ spring constant, $Q$ quality factor and $\omega_{0}$ natural angular frequency. Since the sharpness of the tip correlates with the effective contact area thus the shape of the tip can also influence the results.

Figure 10 presents the histograms obtained on the dissipation maps of the grating and reference area. Compared to the normal distribution of the reference area, with a median around $1.7 \mathrm{pW}$ two distinct peaks can clearly be observed in the histogram of the grating which belong to the valleys and peaks at $1.55 \mathrm{pW}$ and $1.9 \mathrm{pW}$, respectively.

\section{Conclusions}

Two AFM methods, which can be applied for the determination of surface mechanical properties - namely the determination of Young's modulus from contact-mode point-spectroscopy force curves and the determination of tip-surface energy

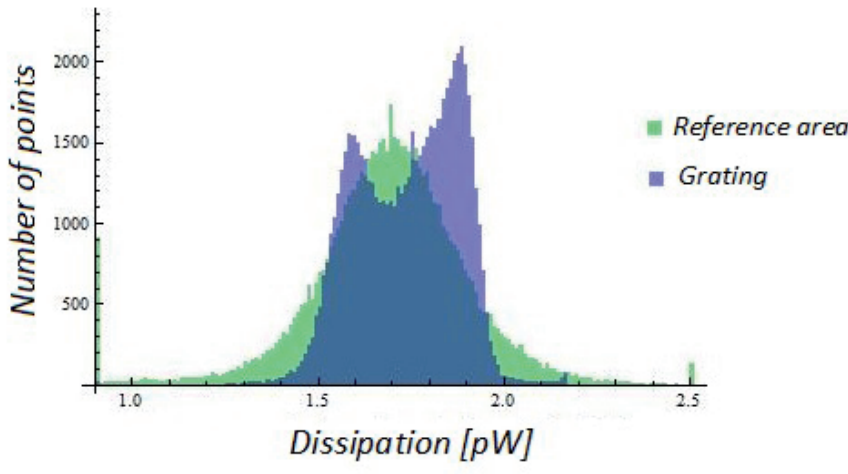

Fig. 10 Histograms obtained on the dissipation maps of the grating and reference area

dissipation through tapping-mode imaging - were discussed in detail and were utilised to investigate the surface properties of an $\mathrm{As}_{20} \mathrm{Se}_{80}$ chalcogenide thin film after laser illumination. The results showed a significant difference in the Young's modulus and tip-surface energy dissipation between the peaks and valleys of the grating. We found the valleys to be softer than the peaks, which can be a possible consequence of the lateral mass transfer which occurred during illumination.

\section{Acknowledgement}

The author would like to acknowledge to István Csarnovics for his contribution to the sample fabrication and to Attila Bonyár for his assistance.

\section{References}

[1] Binnig, G., Quate, C. F., Gerber, Ch. "Atomic force microscopy." Physical Review Letters. 56. pp. 930-933. 1986. DOI: 10.1103/PhysRevLett.56.930

[2] Tseng, A. A. "Advancements and challenges in development of atomic force microscopy for nanofabrication." Nano Today. 6. pp. 493-509. 2011. DOI: 10.1016/j.nantod.2011.08.003

[3] Obermair, C., Kress, M., Wagner, A., Schimmel, T. "Reversible mechano-electrochemical writing of metallic nanostructure with the tip of an atomic force microscope." Beilstein Journal of Nanotechnology. 3. pp. 824-830. 2012. DOI: 10.3762/bjnano.3.92

[4] Cleveland, J. P., Anczykowski, B., Schmid, A. E., Elings, V. B. "Energy dissipation in tapping-mode atomic force microscopy." Applied Physics Letters. 72 (20). pp. 2613-2615. 1998. DOI: 10.1063/1.121434

[5] Anczykowski, B., Gotsmann, B., Fuchs, H., Cleveland, J. P., Elings, V. B. "How to measure energy dissipation in dynamic mode atomic force microscopy." Applied Surface Science. 140. pp. 376-382. 1999. DOI: 10.1016/S0169-4332(98)00558-3

[6] Kikineshi, A., Palyok, V., Shiplyak, M., Szabó, I. A., Beke, D. L. "Photo-induced surface deformation during hologram recording in a-Se films." Journal of Optoelectronics and Advanced Materials. 2 (1). pp. 95-98. 2000.

[7] Codan, B., DelFavero, G., Martinelli, V., Long, C. S., Mestroni, L., Sbaizero, O. "Exploring the elasticity and adhesion behavior of cardiac fibroplasts by atomic force microscopy indentation." Material Science Engineering C, Materials for Biological Applications. 2014. DOI: $10.1016 / \mathrm{j} . \mathrm{msec} .2014 .04 .003$ 
[8] Herzt, H. "Ueber die Berührung fester elastischer Körper." (The contact of solid elastic bodies.) Journal für die reine und angewandte Mathematik. pp. 156-172. 1882. (In German)

[9] Johnson, K. L., Kendall, K., Roberts, A. D. "Surface energy and the contact of elastic solids." Proceedings of the Royal Society of London, Series A. 324. pp. 301-313. 1971. DOI: 10.1098/rspa.1971.0141

[10] Derjaguin, B. V., Muller, V. M., Toporov, Y. P. "Effect of the contact deformation on the adhesion of particles." Journal of Colloid and Interface Science. 53. pp. 314-326. 1975. DOI: 10.1016/0021-9797(75)90018-1

[11] Butt, H. J., Cappella, B., Kappl, M. "Force measurements with the atomic force microscope: Technique, interpretation and applications." Surface Science Report. 59 (1-6). pp. 1-152. 2005. DOI: 10.1016/j.surfrep.2005.08.003

[12] Domke, J., Radmacher, M. "Measuring the elastic properties of thin polymer films with the AFM." Langmuir. 14. pp. 3320-3325. 1998. DOI: $10.1021 / 199713006$

[13] Chang, Y. R., Raghunathan, V. K., Garland, S. P., Morgan, J. T., Russell, P., Murphy, C. J. "Automated AFM force curve analysis for determining elastic modulus of biomaterials and biological samples." Journal of the Mechanical Behavior of Biomedical Materials. 37. pp. 209-218. 2014. DOI: 10.1016/j.jmbbm.2014.05.027

[14] Melzak, K. A., Moreno-Flores, S., Yu, K., Kizhakkedathu, J., TocaHerrera, J. L. "Rationalized Approach to the Determination of Contact Point in Force-Distance Curves: Application to Polymer Brushes in Salt Solutions and in Water." Microscopy Research and Technique. 73. pp. 959-964. 2010. DOI: 10.1002/jemt.20851

[15] Crick, S. L., Yin, F. C. P. "Assessing micromechanical properties of cells with atomic force microscopy: importance of the contact point." Biomechanics and Modeling in Mechanobiology. 6. pp. 199-210. 2007. DOI: 10.1007/s10237-006-0046-x

[16] García, R., Pérez, R."Dynamic atomic force microscopy methods." Surface Science Reports. 47. pp. 197-301. 2002.

DOI: $10.1016 / \mathrm{S} 0167-5729(02) 00077-8$
[17] Martin, M. J., Fathy, H. K., Houston, B. H. "Dynamic simulation of atomic force microscope cantilever oscillating in liquid." Journal of Physics. 104. 044316. 2008. DOI: 10.1063/1.2970154

[18] Lee, S. I., Howell, S. W., Raman, A., Reifenberger, R. "Nonlinear dynamics if microcantilevers in tapping-mode atomic force microscopy: A comparison between theory and experiment." Physical Review B. 66. 115409. 2002. DOI: 10.1103/PhysRevB.66.115409

[19] Cappella, B., Dietler, G. "Force-distance curves by atomic force or microscopy." Surface Science Reports. 34. pp. 1-104. 1999. DOI: 10.1016/S0167-5729(99)00003-5

[20] Gertners, U., Teteris, J. "Surface relief formation in amorphous chalcogenide thin films during holographic recording." Optical Materials. 32. 2010. pp. 807-810. DOI: 10.1016/j.optmat.2010.01.008

[21] Kaganovskii, Yu., Beke, D. L., Charnovych, S., Kökényesi, S., Trunov, M. L. "Inversion of the direction of photo-induced mass transport in $\mathrm{As}_{20} \mathrm{Se}_{80}$ films: Experiment and theory." Journal of Applied Physics. 110. 063502. 2011. DOI: 10.1063/1.3636392

[22] Trunov, M., Lytvyna, P., Takats, V., Charnovich, I., Kökényesi, S. "Direct surface relief formation in $\mathrm{As}_{20} \mathrm{Se}_{80}$ layers." Journal of Optoelectronics and Advanced Materials. 11 (12). pp. 1959-1962. 2009.

[23] Bonyár, A., Molnár, L. M., Harsányi, G. "Localization factor: a new parameter for the quantitative characterization of surface structure with atomic force microscopy (AFM)." MICRON. 43. pp. 305-310. 2012. DOI: 10.1016/j.micron.2011.09.005

[24] Xia, Y., Bigerelle, M., Marteau, J., Mazeran, P. E., Bouvier, S., Iost, A., "Effect of Surface Roughness in the Determination of the Mechanical Properties of Material Using Nanoindentation Test." Scanning. 36. pp. 134-149. 2014. DOI: 10.1002/sca.21111

[25] Cheng, W., Dunn, P. F., Brach, R. M. "Surface roughness effects on microparticle adhesion." The Journal of Adhesion. 78. pp. 929-965. 2002. 